SHORT REPORT

\title{
Mortality and morbidity surrounding coronary artery bypass surgery and the public presentation of risk
}

\author{
R M Martin, D Gunnell, K R Karsch, S Frankel
}

J Epidemiol Community Health 2002;56:430-431

$\mathrm{D}$ eaths of patients on the waiting list for coronary artery bypass surgery are often presented by the media to the public and politicians as evidence of the remediable failure of health care.' The assumption that many lives would be saved by more timely intervention is over-simplistic and takes no account of three important observations. Firstly, for approximately one third of low risk patients the principal reason for performing coronary artery bypass procedures is for the relief of angina, rather than mortality prevention. ${ }^{2}$ Secondly, people on waiting lists inevitably have widespread vascular disease and the atherosclerotic lesions that cause sudden death may not be the same as those for which the patient has been listed for relief of angina. ${ }^{3}$ Lastly, operative death rates for elective surgery of $1 \%-2 \%{ }^{4}$ mean a proportion of patients die as a result of the procedure itself.

We have compared the mortality and myocardial infarction risk of those waiting for coronary artery bypass surgery with those undergoing this procedure using routine and published data. The aim is to estimate the numbers of deaths and myocardial infarctions in each time period to determine whether, at a population level, the frequent attention drawn to adverse events while on a waiting list should be more equally balanced by consideration of the risk of perioperative and postoperative adverse events.

\section{SUBJECTS, METHODS, AND RESULTS}

Approximately 15000 waiting list or booked admissions received a coronary artery bypass graft in an NHS hospital in England in 1998/99 (Department of Health, Hospital Episodes Statistics, personal communication). Waiting time is defined as time on the waiting list from decision to admit until admission for the operation. Based on the waiting time of these 15000 people and published estimates for the incidence of death (range: 3.4 to 4.1 per 100 patient years ${ }^{56}$ ) and myocardial infarctions (4.2 per 100 patient years ${ }^{6}$ ) while on waiting lists we computed that there are approximately 280 to 330 waiting list deaths and 340 myocardial infarctions each year in England (table 1). Using mortality data from two recent sources we estimated that there are between $285^{4}$ and $390^{8}$ deaths within six months after operative intervention each year in England. Our estimated six month postoperative myocardial infarction rate $(5 \%)$ was derived from the Randomised Intervention Treatment of Angina (RITA) trial ${ }^{7}$, giving an estimated 750 events after surgery per year in England. Both these figures are conservative estimates of risk as the study populations were low risk patients.

\section{DISCUSSION}

Our results suggest that levels of mortality experienced within six months of coronary artery bypass surgery are similar to those experienced while waiting for the procedure. The estimated number of patients suffering a myocardial infarction during their wait for treatment was markedly lower than the number of events within six months of coronary artery bypass surgery. Public, political, and indeed clinicians', attention tends to highlight the former as evidence of unacceptable failure in the health care system, while drawing little attention to the potential disbenefits of this procedure.

There are limitations to the conclusions that can be drawn from this study. Firstly, the comparisons we have made here are not an attempt to estimate either the effectiveness of coronary artery bypass surgery, or the relative balance of benefits and risks associated with early intervention for particular patients. There is clear evidence from randomised controlled trials that an initial strategy of coronary artery bypass surgery is associated, in the long term, with reduced mortality rates in patients with significant left main-stem stenosis, three vessel disease and proximal left anterior descending artery disease. ${ }^{2}$ However, it should be remembered that, on average, coronary

Table 1 Estimated numbers of deaths and myocardial infarctions in England among those waiting for coronary artery bypass graft surgery and postoperatively

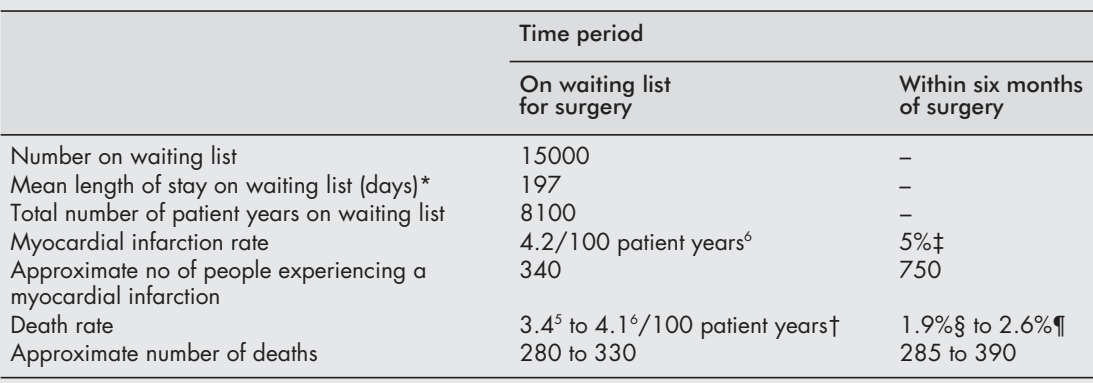

* Source: Department of Health, Hospital Episodes Statistics (personal communication). †Sources: Seddon et $a^{5}$ and Billing et $a^{k}$ (this latter paper reports two analyses of waiting list mortality-one of a single surgeon's experience and based on only 12 deaths in 496 patients awaiting bypass surgery, the other based on 57 deaths in 3453 patients awaiting cardiac surgery; we used the latter estimate (4.1 per 100 patient years) in the estimate given above. $¥$ Source: Rita trial participants ${ }^{7}$ an estimate of the six-month myocardial infarction rate was derived from figure 2 of this paper. §Source: Brorsson et al. ${ }^{4}$ qUK Cardiac Surgical Register. ${ }^{8}$ 
artery bypass surgery prolongs life by only 4.26 months. ${ }^{2}$ Secondly, the six month postoperative period analysed here accentuates mortality risk associated with the procedure itself; for people who would benefit prognostically from surgery, the total risk faced is the sum of the risk while waiting plus the postoperative risk, which would be greater than if those people had not waited at all.

There are also limitations to the data sources used in this study. The data from Billing et al, used to compute waiting list death and myocardial infarction rates, were collected between 1988-93 in a single hospital in England with a mean wait of 147 days. ${ }^{6}$ As bypass surgery was far less frequently performed at that time, the patients may have had more severe disease making them at higher risk and leading, therefore, to an overestimate of waiting list events. However, the results were close to those estimated from more recent studies. ${ }^{5}$ In the RITA trial, which recruited between 1988-1991, only 3\% of subjects with angiographically proven coronary artery disease were randomised. ${ }^{7}$ The generalisability of the postoperative myocardial infarction rate to the current national case mix is therefore uncertain. The study by Brorsson $e t$ al ${ }^{4}$ was restricted to subjects with chronic stable angina undergoing coronary artery bypass graft for the first time in seven hospitals in Sweden. The postoperative mortality rate is lower than the $2.6 \%$ reported in the UK Cardiac Surgical Register, 1998, for coronary artery bypass surgery. ${ }^{8}$

We accept that waiting lists are likely to adversely affect those with vessel disease for whom trial evidence suggests mortality advantages from coronary surgery and those whose daily life is impaired by cardiac symptoms despite maximum medical treatment. Instead our study highlights the trade off between procedure related mortality and future prognosis and quality of life that doctors and patients have to consider in individual cases. The relative benefits and risks are likely to be more finely balanced for low risk patients or the elderly, for whom trial evidence of benefit is scant. The risk/benefit ratio in older age groups is still unknown as in the original trials of coronary bypass surgery only $7 \%$ of subjects were over 60 years. $^{2}$ In 1998 a total of $68.2 \%$ of elective coronary bypass grafts were for people aged 60 years or over (Hospital Episodes Statistics, personal communication), a group who are at greater risk of perioperative mortality and postoperative morbidity.

This study does not condone the existence of waiting lists. However, it suggests that a more balanced portrayal of the true risks of operative treatment will be important in developing a greater public understanding of the relative merits of prevention, medical management, and surgical measures in the reduction of the morbidity and mortality attributable to coronary heart disease. The use of expert panel ratings of appropriateness, which are a reflection of net expected benefit and harm, and which have been validated against clinical outcomes, ${ }^{9}$ offer a more appropriate routine decision making tool in improving the quality of care, than the current focus on waiting list targets.

\section{Key points}

- Deaths while waiting for coronary artery bypass surgery are often presented as evidence of the remediable failure of health care.

- We found that mortality rates within six months of bypass surgery are similar to those experienced while waiting for the procedure.

- A more balanced portrayal of operative benefits and risks is required to develop public understanding of the relative merits of prevention, medical management, and surgery in the reduction of coronary heart disease mortality.

\section{Contributors}

The paper was originally conceived and drafted by RM, DG and SF. KK provided clinical input and RM, DG, SF and KK contributed to the redrafting and editing of the final text. HES data were made available by the Department of Health to the authors courtesy of the HES National Service Framework project (Professor Shah Ebrahim and colleagues), funded by a South and West Regional project R\&D grant.

\section{Authors' affiliations}

R M Martin, D Gunnell, S Frankel, Department of Social Medicine, University of Bristol, Canynge Hall, Whiteladies Road, Bristol BS8 2PR, UK

K R Karsch, Bristol Heart Institute, University of Bristol

Funding: none.

Conflicts of interest: none.

Correspondence to: Professor S Frankel; stephen.frankel@bris.ac.uk

Accepted for publication 7 November 2001

\section{REFERENCES}

1 Hall C. NHS heart surgery delays 'may kill 500 patients a year'. London: Telegraph Group Limited, 1999.

2 Yusuf S, Zucker D, Peduzzi P, et al. Effect of coronary artery bypass graft surgery on survival: overview of 10 year results from randomised controlled trials by the Coronary Artery Bypass Graft Surgery Triallists Collaboration. Lancet 1994;344:563-70.

3 Danchin N. Is myocardial revascularisation for tight coronary stenoses always right? Lancet 1993;342:224-5.

4 Brorsson B, Lindvall B, Bernstein SJ, et al. CABG in chronic stable angina pectoris patients: indications and outcomes (SECOR/SBU). Swedish Societies for Cardiology, Thoracic Radiology and Thoracic Surgery/Swedish Council for Technology Assessment in Health Care. Eur Jurgery/Swedish Council for Technology A

5 Seddon ME, French JK, Amos DJ, et al. Waiting times and prioritisation for coronary artery bypass surgery in New Zealand. Heart 1999;81:586-92.

6 Billing JS, Arifi AA, Sharples LD, et al. Heart surgery in UK patients: planned care or crisis management? Lancet 1996;347:540-1.

7 RITA trial participants. Coronary angioplasty versus coronary artery bypass surgery: the Randomised Intervention Treatment of Angina (RITA) trial. Lancet 1993;341:573-80.

8 Keogh BE, Klinsman R. National adult cardiac surgical database report, 1998. London: The Society of Cardiothoracic Surgeons of Great Britain and Ireland, 1999.

9 Hemingway H, Crook AM, Feder G, et al. Underuse of coronary revascularisation procedures in patients considered appropriate candidates for revascularisation. NEJM 2001;344:645-54. 